\title{
Managing bathymetric data in a hydrographic survey company and making the data accessible to clients
}

\author{
Duncan Mallace \\ Tim Kearns 2
}

\author{
${ }^{1}$ NetSurvey Limited, $2 A$ Banbury Office Village, Noral Way, Banbury, OX16 2SB, UKａncan@netsurvey.co.uk \\ 2Esri Inc., 380 New York St, Redlands, CA 92373-810o, USAｔkearns@esri.com
}

Topic F: careful marine planning

\begin{abstract}
As data volumes increase, managing the large hydrographic datasets is not limited to the world's hydrographic offices. The hydrographic survey companies contracted to carry out the surveys need to be able to store their data in an easily accessible, spatial and quick manner. They also need to be able to serve their data to clients via The Cloud. This demand is being increasingly seen across all the survey sectors, from Oil and Gas through to Offshore Renewables, Hydrography through to Coastal Surveys.

MMT Group (NetSurvey and MMT combined) have almost 1 Petabyte of survey data that was stored, like most survey companies, in a flat, file server-based setup. Trying to find one particular survey was particular challenging and trying to find consecutive surveys was almost impossible. NetSurvey and MMT have performed hydrographic surveys for the Maritime \& Coastguard Agency (MCA) in the UK, the Mareano Project for the Norwegian Hydrographic Service and also the Swedish and Finnish Maritime Administrations. The MCA surveys are both large in area and, for the Routine Re-Survey Contract, in time (over seven years). We wanted a system that would not only store bathymetry but also sediment types, digital video, survey reports and sub-bottom data. The Esri Bathymetric Information System (BIS) is a database specifically designed for this type of requirement and is accessed through the well-known and easily used Esri ArcGIS software. The Esri products also have the capability to serve the data either publicly or privately (to select clients and internally) via a simple web interface.
\end{abstract}

\section{Introduction}

For a spatially knowledgeable industry we have a very flat approach to storing our data.

MMT Group conducts high resolution surveys in the fields of Hydrography, Oil \& Gas Exploration, Offshore Renewable Energy and Cable industries. As the technology improves, so do the data quantities. Companies keep adding more storage capacity to their servers but this increase in the number and size of the servers creates a new problem. The data might be grouped by year or industry, but most likely it is collated into folders named after the project number, Hydrographic Instruction, Date or maybe all three. How do you go about finding the surveys you performed off East Anglia for instance in the last three years? If we bid for a survey tender it is very useful to be able to bring in the bounds of the survey area and see if it is adjacent or nearby to a previous survey. Metrics such as weather downtime and seabed sediment type can then be used to streamline the tender. Spatially viewing the datasets allows us to realise where datasets are. What we do not want to do though is duplicate our already huge datasets.

In nearly all of the survey sectors that we work in a GIS deliverable is becoming more common. In the vast majority of client's specifications, that GIS deliverable is an Esri ArcGIS geodatabase. Sometimes clients have their own geodatabase format to use (for example NordStream) but with ArcGIS 10.1 the use of data models as templates for deliverables is becoming more common. Examples of this are the Seabed Survey Data Model (developed by the OGP Geomatics Committee (formerly EPSG) and the ArcMarine datamodel for Habitat Surveys. Obviously it is much easier to produce an ArcGIS deliverable when you can access all your data from within ArcGIS products.

Another common factor between the different sectors is that the survey area size is becoming larger and the resolution of the final product is becoming higher. This stretches the limits of computing hardware to the maximum and one of the reasons for adopting the Esri Maritime Bathymetry solution is that it makes it possible to combine very large, very high resolution datasets and geospatial tools (like contouring and slope calculations) across the whole dataset and not just on individual elements. This saves time and complexity.

This extended abstract explains what the BIS is, how the BIS was built and setup at MMT Group and how we use it to produce products and future plans. 


\section{Bathymetric Information System}

The Bathymetric Information System (BIS) is a commercial enterprise GIS system for the management and deployment of bathymetric data, products and related information. It includes specialised schemas, metadata engine, functionality for ingestion of data and the ability to query, filter and compose continuous bathymetric surfaces based upon a user specified model. As well, the BIS supports workflows, integration with complementary applications, and the ability to generate image and map services within a service-oriented architecture and a commercially available web application for query and dissemination.

ArcGIS for Maritime: Bathymetry can be used for;

- Managing bathymetric data from a central geodatabase

- Storing and querying gridded bathymetric datasets and creating custom surfaces from them

- Creating and managing collections of bathymetric datasets

- Managing metadata for Bathymetric Attributed Grids (BAGs) from the Open Navigation Surface project for collections of datasets, or managing any custom metadata associated with the datasets

- Creating and storing custom bathymetric surfaces

- Extracting depth values from bathymetric surfaces

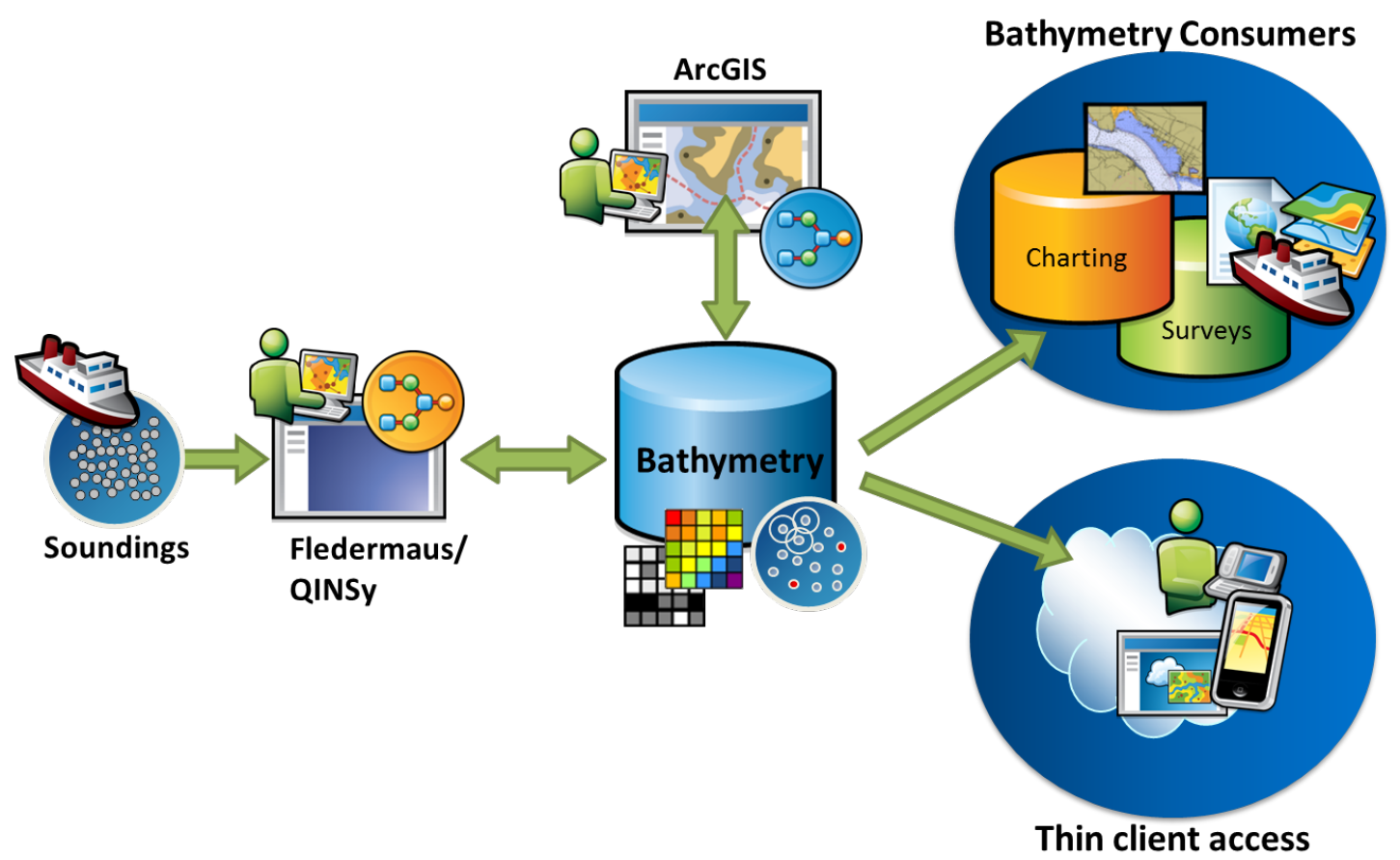

Figure 1: The Esri/QPS Bathymetric Information System is designed to handle large volumes of vector and gridded data that leverages an enterprise GIS platform. This includes integration with spatial and non-spatial databases, production environments and cloud computing.

The primary objectives of such a system is to manage post-processed soundings and depths, ancillary information about the hydrographic survey (report, survey area, tidal adjustment reports, sound velocity cast reports, navigation lines, other supporting information), metadata about surveys, vector and gridded data and the storage and dissemination of bathymetric gridded data. An enterprise system must also be able to easily communicate in a service-oriented architecture via published feature and map services, integration with other geospatially enabled databases and consuming other data feeds through mash-ups and crowd sourcing.

The main architectural components of the BIS are common in the GIS industry. Complementary technology, QPS Fledermaus \& QINSy, serves as the primary ingest engine for survey related content in both vector and gridded data format. Reading and writing directly to the Esri geodatabase allows for a seamless flow of high density survey data through a quality control and visualisation application before it's appended to a central geodatabase. Once the 
bathymetric data is processed and cleaned it is managed from a central geodatabase by the Esri mosaic dataset data type. The mosaic dataset allows for bathymetric data to remain unaltered and unduplicated.

Using a bathymetric geodatabase brings many advantages to maritime GIS users. They can maintain a historical archive for comparing legacy survey data with modern data and updated charts. They can also leverage bathymetric data by using it with other databases for a wide range of purposes, such as oceanography, coastal zone management, and meteorology that require seafloor information. In addition, users can easily edit their nautical products based on new and updated bathymetric information.

\section{Building the BIS}

Figure 2 below shows the fileserver setup in NetSurvey's office and will be common to most companies as to how they store their survey data. The storage keeps getting added to as the surveys come in and within each storage system the data is stored in folders named by project number. This is a good way of storing the data and absolutely essential for efficient file management. However, it is not a good way to view the data or to retrieve it.

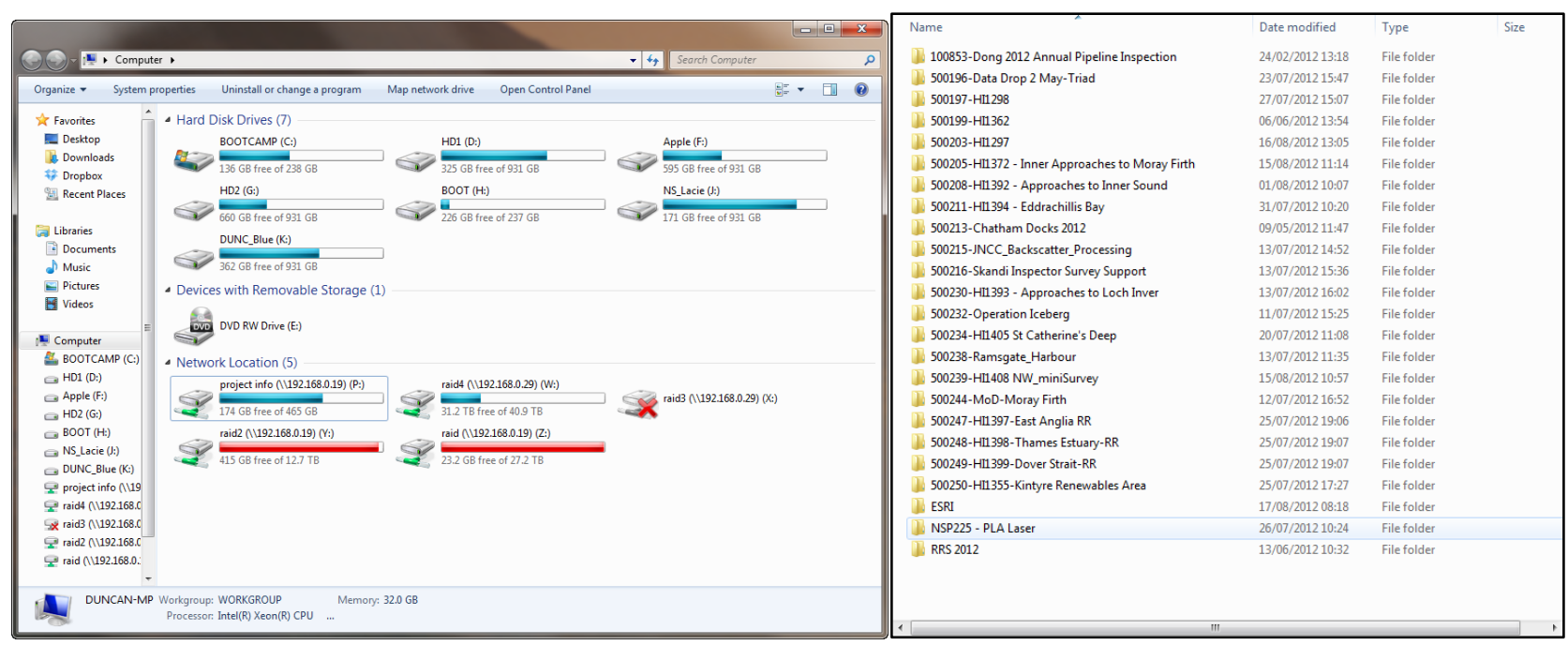

Figure 2: NetSurvey's folder example folder structure (left) and the RAID 4 structure (right).

One of the main reasons for MMT Group choosing the Esri Bathymetric Information System rather than some rival solutions is that it does not replicate the data by ingesting it into the geodatabase. Rather it maintains a link to the data within our existing file server network. Our completed surveys are archived in the Bathymetric Attributed Grid Format (BAG). The BAG is the highest resolution digital terrain model that can be obtained from the data and is normally the client's deliverable cell size as well. We create the BAG from within our QPS Fledermaus application (fig 3 below). It is a simple one button push and then a metadata field editor where additional information can be added. The fields are limited, as it is part of the BAG format, but more metadata can be added as External Metadata within ArcGIS later. 


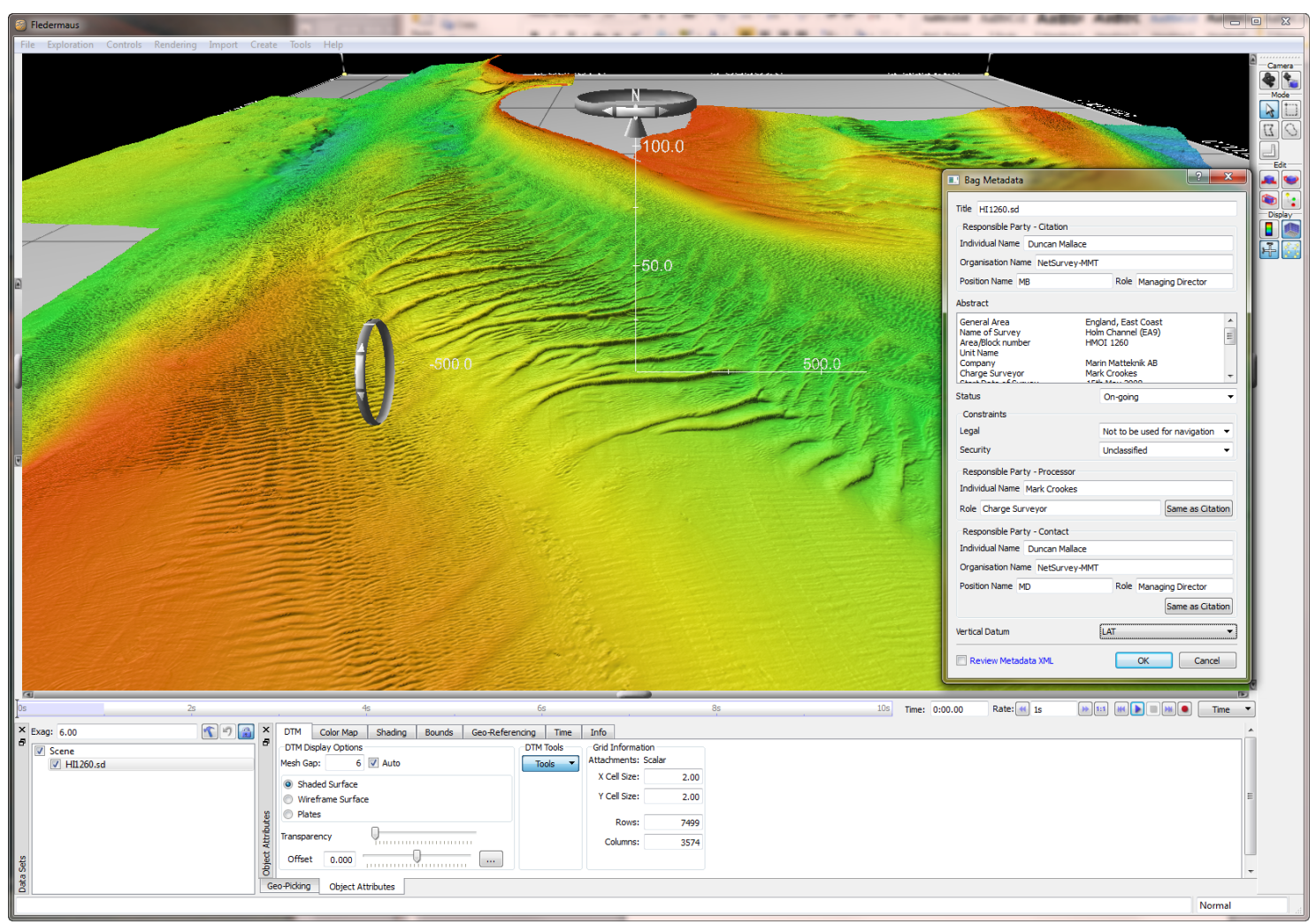

Figure 3: BAG creation in Fledermaus

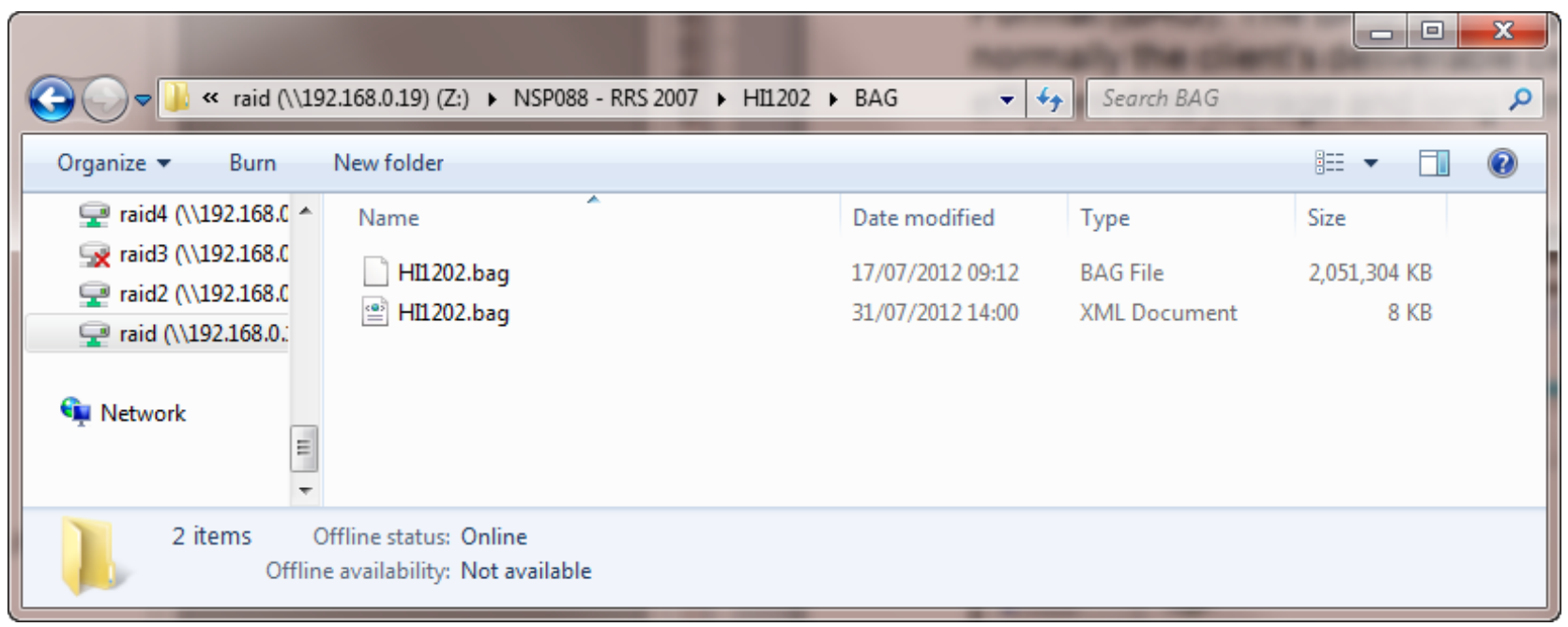

Figure 4: Image showing BAG example within file server

The BAG format is open source and compressed so it makes for efficient data storage and long term viability. It also contains information pertaining to the accuracy of the data as an Uncertainty layer.

MMT Group wanted to have a system that was easy to use, didn't require any special server structure and allowed for future expansion and progression. The BIS can be run as a standalone File Geodatabase or as an Enterprise ArcSDE Geodatabase. One of the benefits of ArcGIS is that it can run on any number of different types of database, so depending on your company's skill set you can choose to run it on Microsoft SQL, PostgreSQL, Oracle, etc.. or as a humble file. Esri's spatial toolset runs on top of all of these. The other packages that MMT Group looked at all had to run on a dedicated Oracle or PostgreSQL server which involved much more back office IT resources and skill set.

Creating the BIS was quite simple. It is all performed from the ArcMap interface, which is familiar to anyone who has used ArcGIS as this is the application where everything is built. All the tools available with the Bathymetry Solution are accessible from the Bathymetry toolbar. The Create BIS button is selected and the dialogue box in Figure 5 appears, giving the user the option to name, add metadata and select the type of geodatabase to use for the BIS. This then creates an empty BIS to which bathymetric data can be added. 


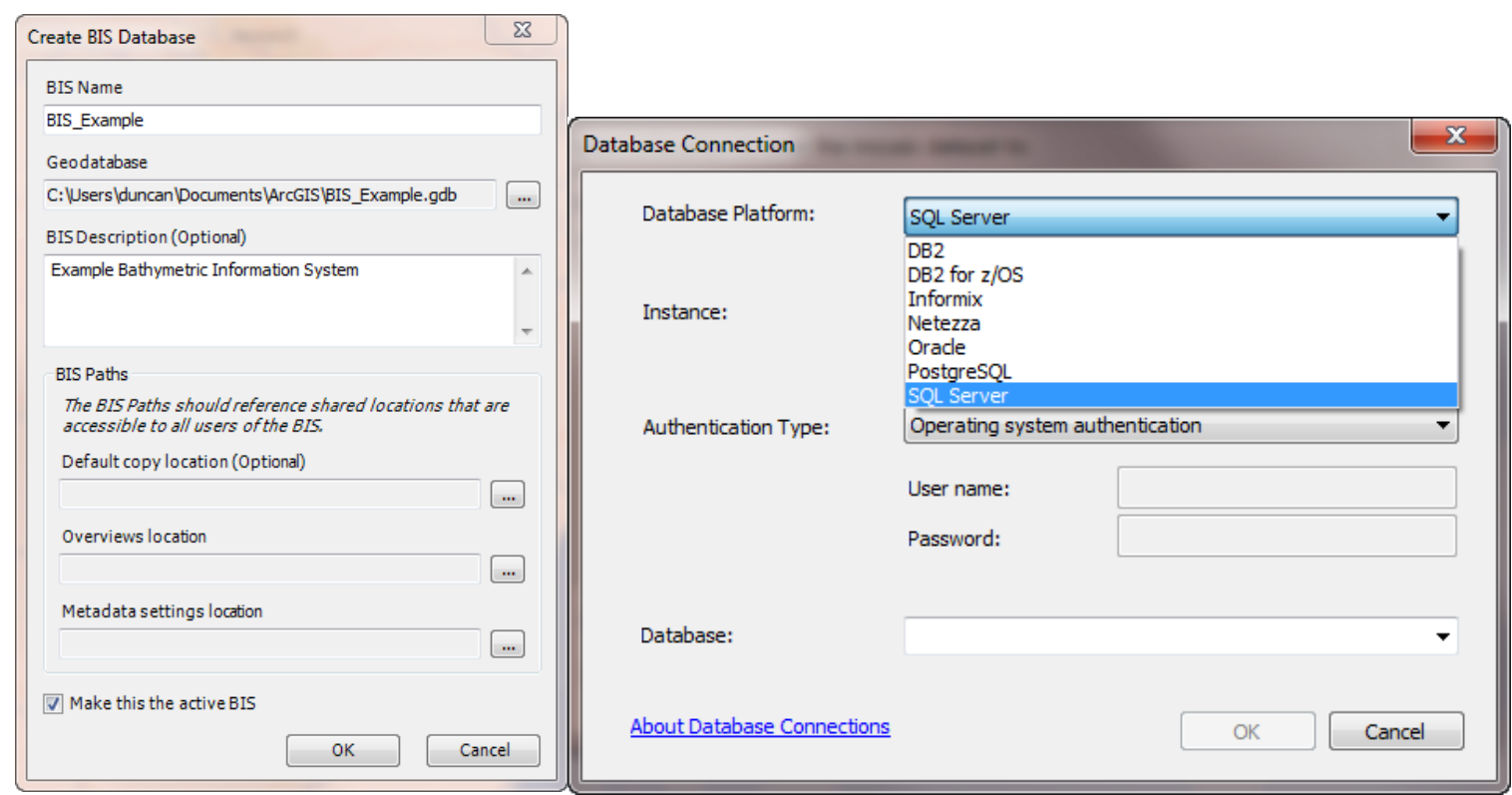

Figure 5: Images showing the dialogue boxes to create a BIS

Once established we then created some collections within the database. A collection is a set of associated surveys. We created collections based on specific survey programmes, geographical areas and specific projects. This doesn't limit the functionality in any way but allows for quickly retrieving data sets and also for adding additional metadata to the collections. In Figure 6 you can see that we have a collection based on our surveys around the Orkney Islands. The metadata that includes overviews of the individual surveys can be viewed and the individual metadata for a particular survey can be seen.

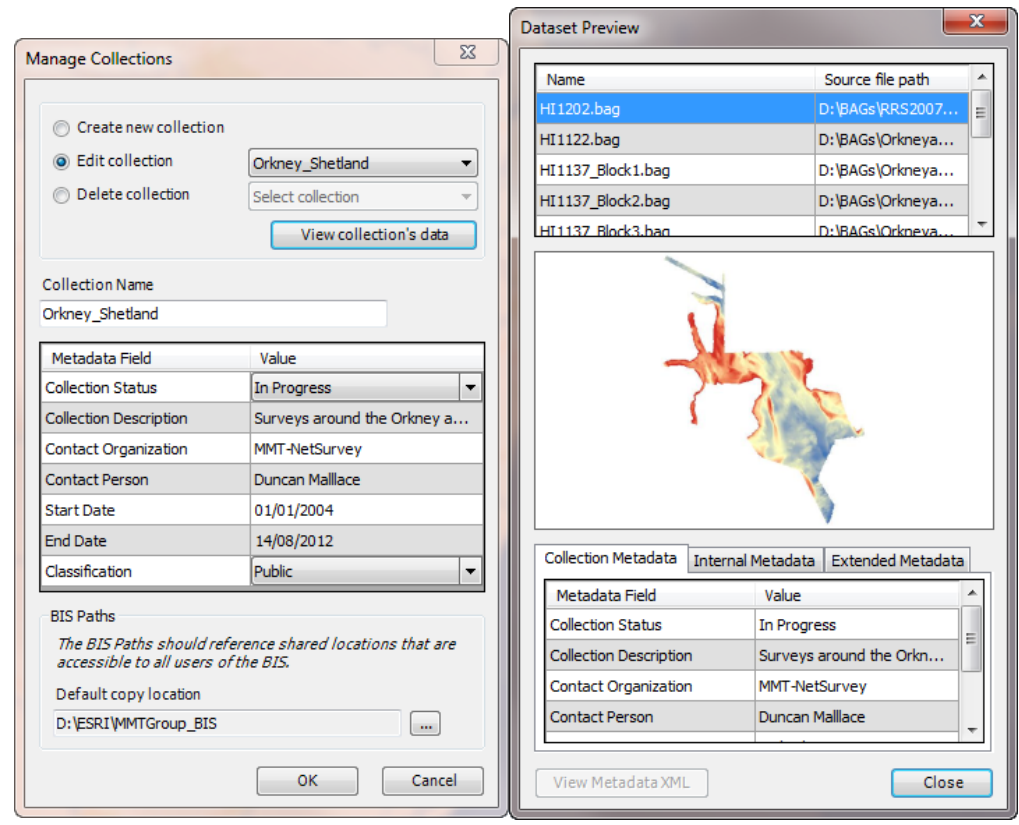

Figure 6: Images showing BIS Collections and a Collection's dataset

Once the collections have been created the BAG that is stored on the storage server can be linked into the BIS. One of the main advantages of the BIS is that the geodatabase can leverage Esri's mosaic dataset data type, which links gridded data stored on-disk to its supporting tables in the BIS geodatabase. This data structure eliminates the need to duplicate data storage on-disk and in a database; with the Bathymetry extension, gridded surface data is stored only once on-disk and is accessed through the mosaic dataset through Bathymetry tools. When we import a BAG into the BIS it automatically contains the Internal Metadata that was input when we created the BAG. The Collection Metadata is associated with the BAG when we choose the collection to add it in to and we also add in additional metadata for the vessel name, type of equipment, IHO order and specific sensor. All of these various metadata fields can be used as a basis for rules when it comes to creating a continuous surface combing these BAGS (figure 7). 


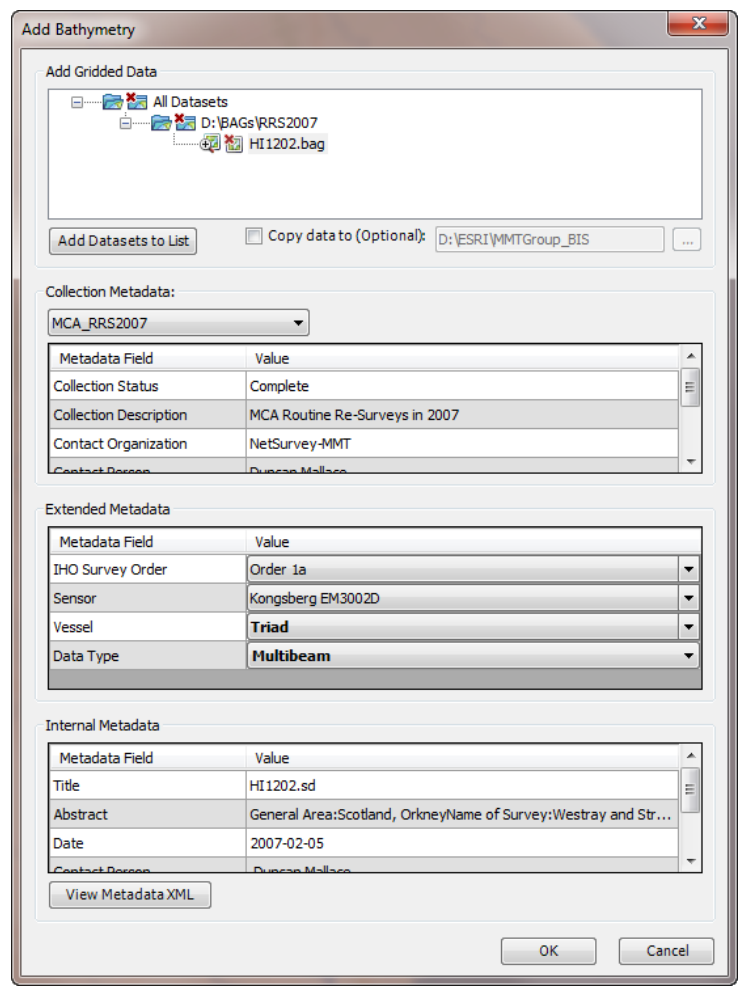

Figure 7: Adding a BAG to the BIS

\section{Using the BIS}

With the data in the BIS it is now possible to explore areas, create continuous surfaces, run analytical tools on the data and bring the data into $3 \mathrm{D}$ visualisation tools, such as QPS Fledermaus.

One of the main reasons for choosing the BIS was to view our surveys spatially. We have used the Esri Ocean Basemap to provide background information and for specific areas we have added in the S57 cells too. To view the surveys that we have performed in Morecambe Bay is as simple as zooming into the area on the Base Map and showing all surveys within the bounds of the screen (figure 8).

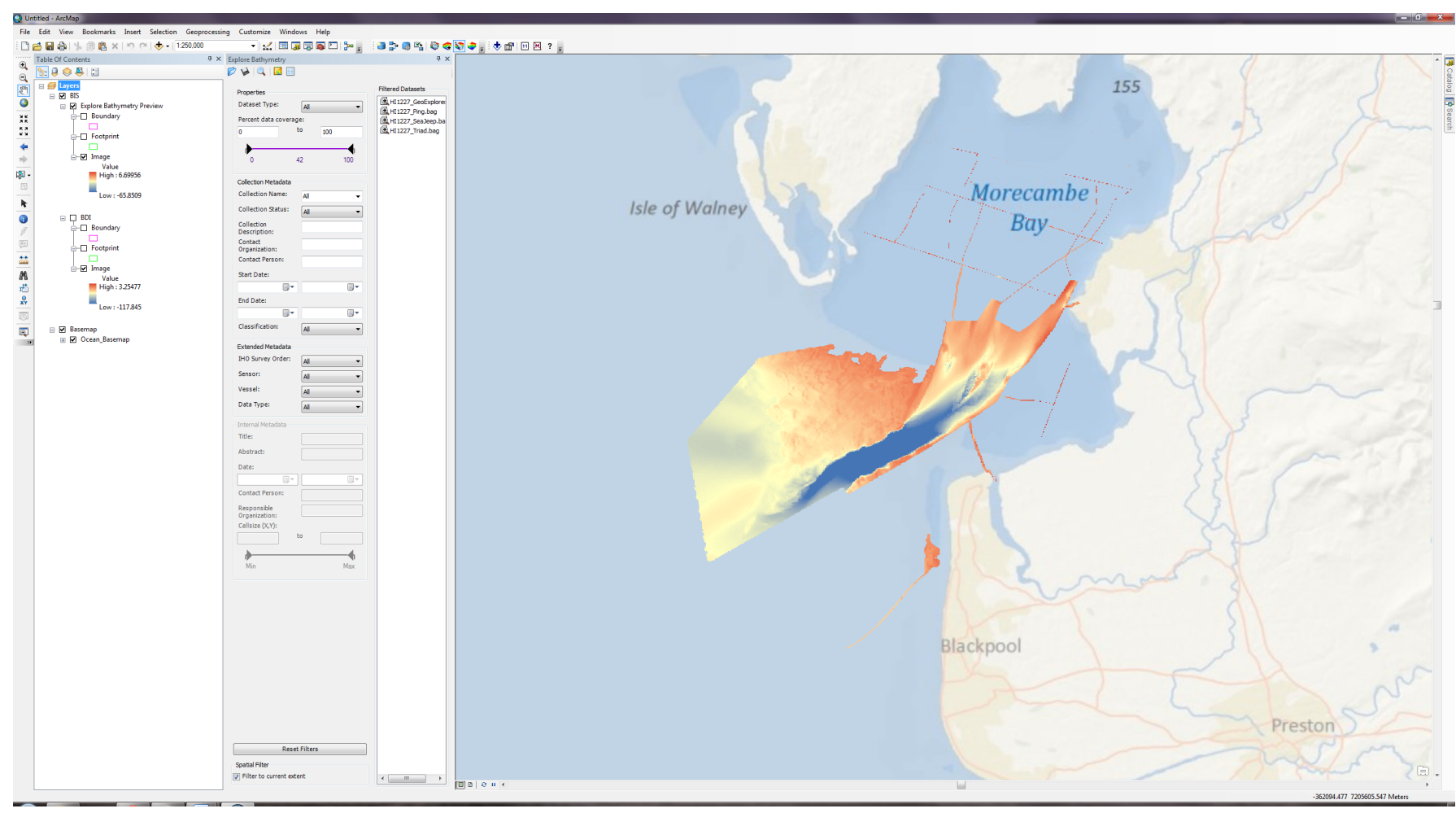

Figure 8: Viewing surveys within certain bounds 
This can be further narrowed down to show a specific vessel's data or types of data. In figure 9 just the surveyed area covered by our survey vessel Ping is shown. This performed just by simple drop down lists containing the different metadata headers.

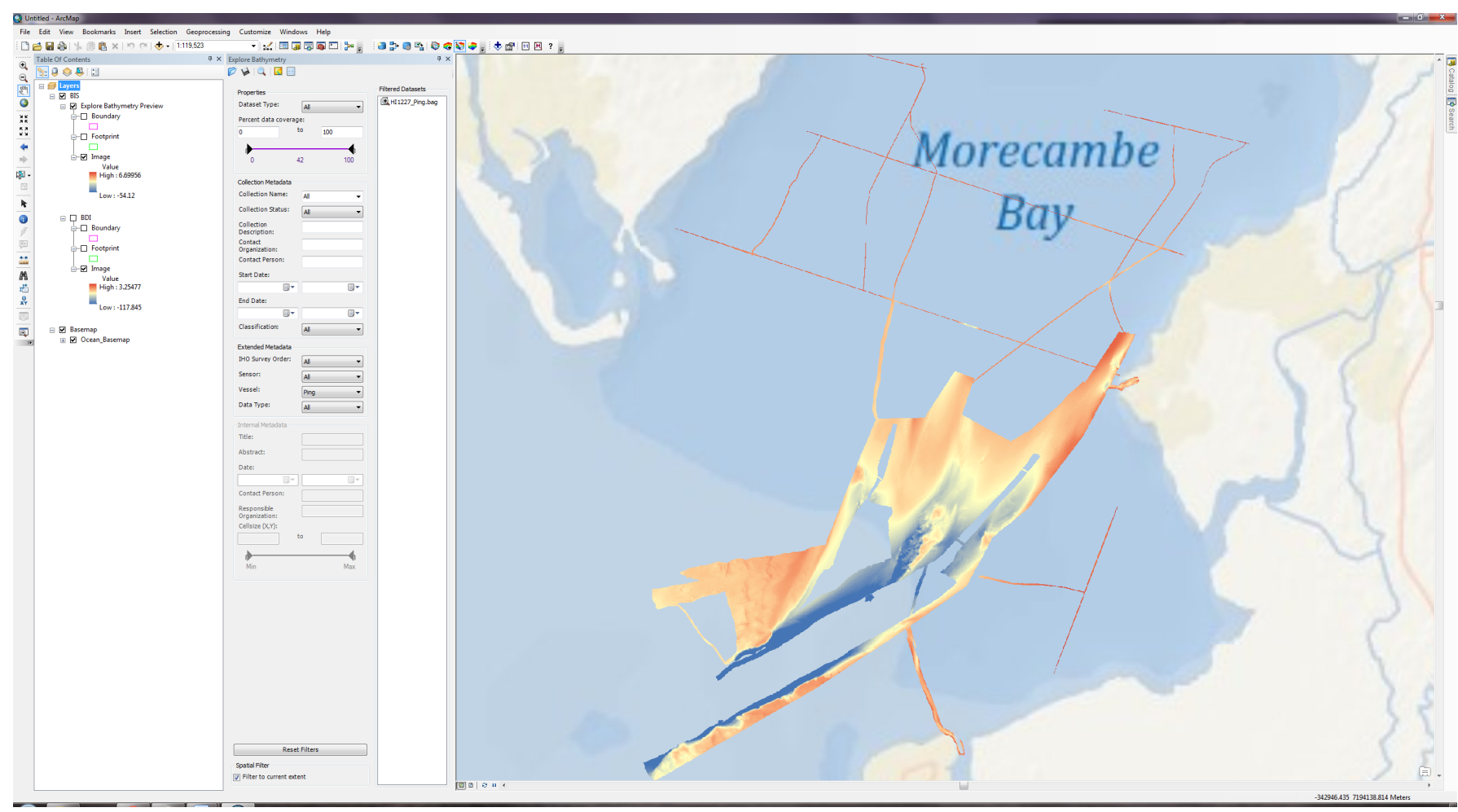

Figure 9: SV Ping's data shown

Being able to view previous surveys is really useful for tendering purposes. Figure 10 shows the surveys in our BIS around the Orkneys. The images of the surveys are shown as mosaic datasets which have pyramid tiles, so as you zoom in the resolution increased. Within this dataset shown we have digital terrain models at $1 \mathrm{~m}, 2 \mathrm{~m}$ and $5 \mathrm{~m}$ resolution, there is no single cell size required and the cell sizes are determined by the original BAG's cell size.

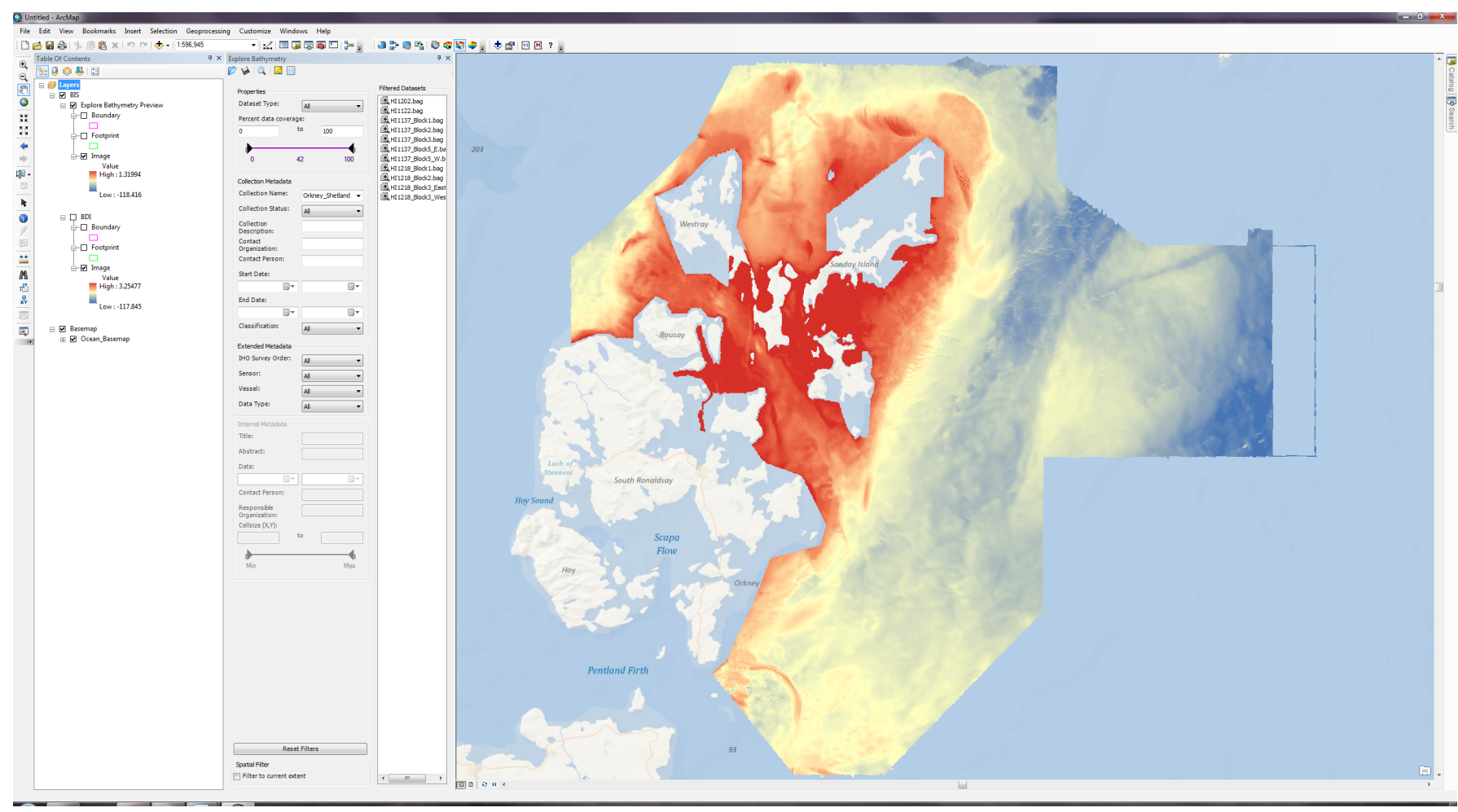

Figure 10: View of different survey BAGs around the Orkney Islands 
I took the Esri shapefile supplied with the tender and loaded it into ArcMap. When you zoom to the shapefile extents you see what survey data is adjacent or overlaps. By identifying the survey we could then drill down into the metadata to determine the time of year the survey took place from which we could analyse the potential weather downtime, from our own actual data for input into the tender calculations. The Bing Maps overlay is used in Figure 11 to provide additional information.

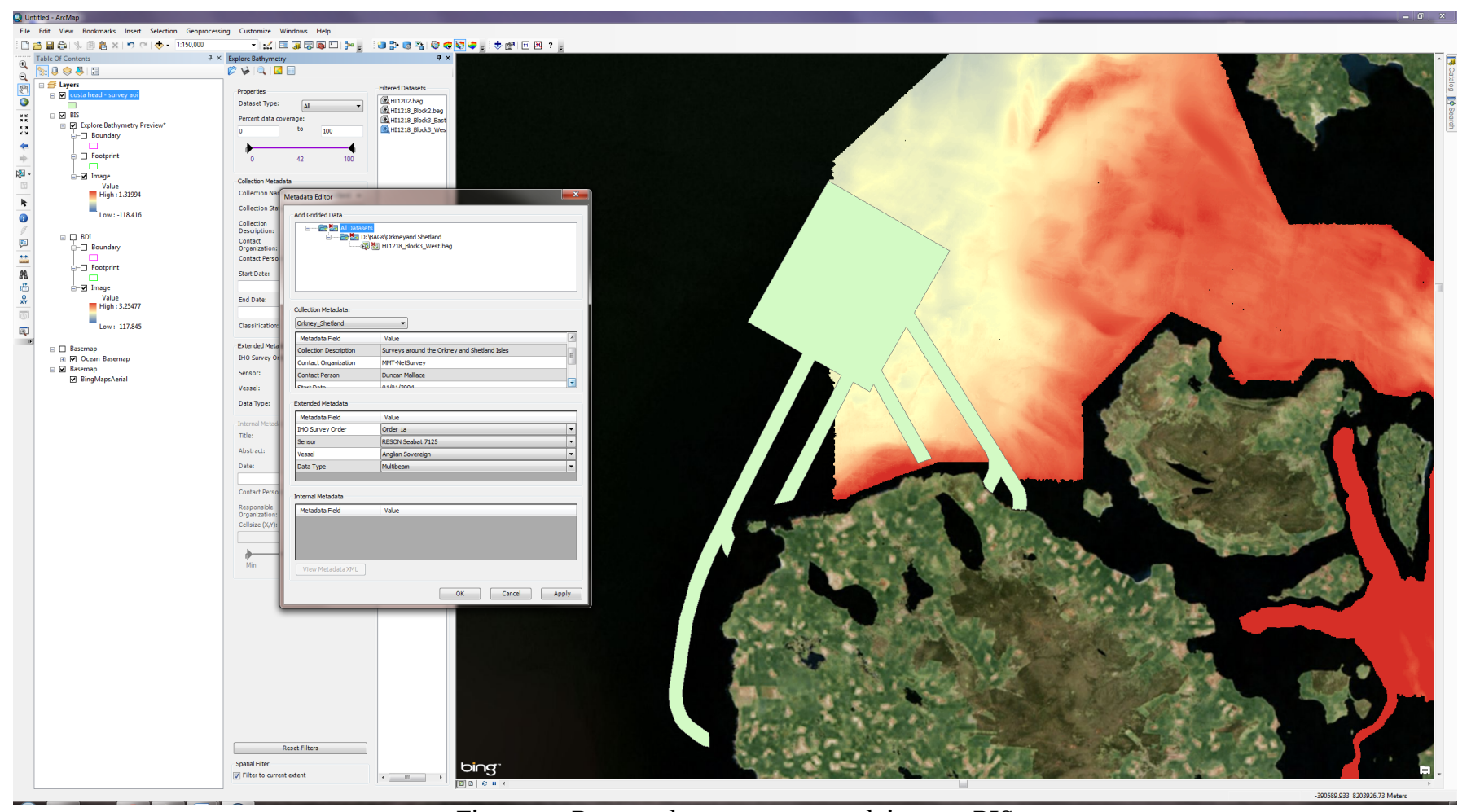

Figure 11: Proposed survey area overlain onto BIS

It would have course been possible to go into our fileserver, take an educated guess as to which survey it was and by a process of trial and error determine the survey, but this would have taken much longer. Provided we had a digital terrain model in a format that could be used easily with a shape file, we could then combine the datasets as above, however, if it was only the soundings that were available then a digital terrain model would have had to be created, potentially taking hours of time to produce.

\section{Creating and using Custom Bathymetric Surfaces}

One of the very best features of the BIS is its ability to combine different digital terrain models together into one seamless surface, from which spatial tools such as contouring and sounding selection can be run. Even with modern computing hardware, the size of the individual surveys is huge and can stress even the very best computers. Using the Esri mosaic technology, a huge surface can be built that links together the BAGs rather than creating an actual file. A mosaic dataset is a collection of raster datasets stored as a catalogue and viewed or accessed as a single mosaicked image or individual images (rasters). These collections can be extremely large both in total file size and number of datasets. The raster datasets in a mosaic dataset can remain in their native format on disk or exist in the geodatabase. The metadata can be managed within the BAGs record as well as attributes in the attribute table. Storing metadata as attributes enables data to be managed easily as well as enabling fast queries to enable selections.

The data can even be completely or partially overlapping but be captured over different dates. The mosaic dataset is an ideal dataset for storing temporal data. You can query the mosaic dataset for the surveys you need based on time or dates, equipment or vessel or any other custom metadata field.

Mosaic datasets are not limited to one particular type of raster data. You can add raster data in different projections, resolutions, pixel depths, and number of bands. Overviews (like pyramids) can be generated for the entire data collection. This allows for faster viewing of the data and allows you to easily serve these datasets. There are also many additional properties for viewing, including setting a mosaicking method, that make these datasets unique and functional in many situations. You can also query a mosaic dataset based on your spatial and non- 
spatial query constraints. The results of that query can be a set of images that you could process one by one, or it could be a dynamically generated mosaicked image.

At MMT Group we use these custom bathymetric surfaces to produce the deliverables required by Oil \& Gas and Renewable Energy companies. Typically and especially with pipeline and cable route surveys, traditional raster technology requires rectangular grids to be created which end up being very large files and sometimes too large for computer memory, so the data is subdivided into sections where the same actions have to be repeated for each block. This creates much longer data processing times than just performing the operation once and can lead to quality assurance issues with features being offset at joins and other cosmetic effects. A single contour file can, for instance, be referenced/linked into multiple alignment sheets. So rather than producing contours from blocks of survey data we combine all the areas together into one Custom Bathymetric Surface in ArcGIS using the Compose surface option. This allows us to specify a rule or just to combine all the datasets in one collection. Once they are combined into one "Surface" the contouring and other functions like long profiles and cross profiles can be generated from this combined surface. Figure 12 to 14 show the steps in combining surfaces, running contours and then viewing the result for the surveys in the Orkneys. First the continuous surface is setup by selecting a rule that says that if there is overlapping data, the survey that is most recent has priority.

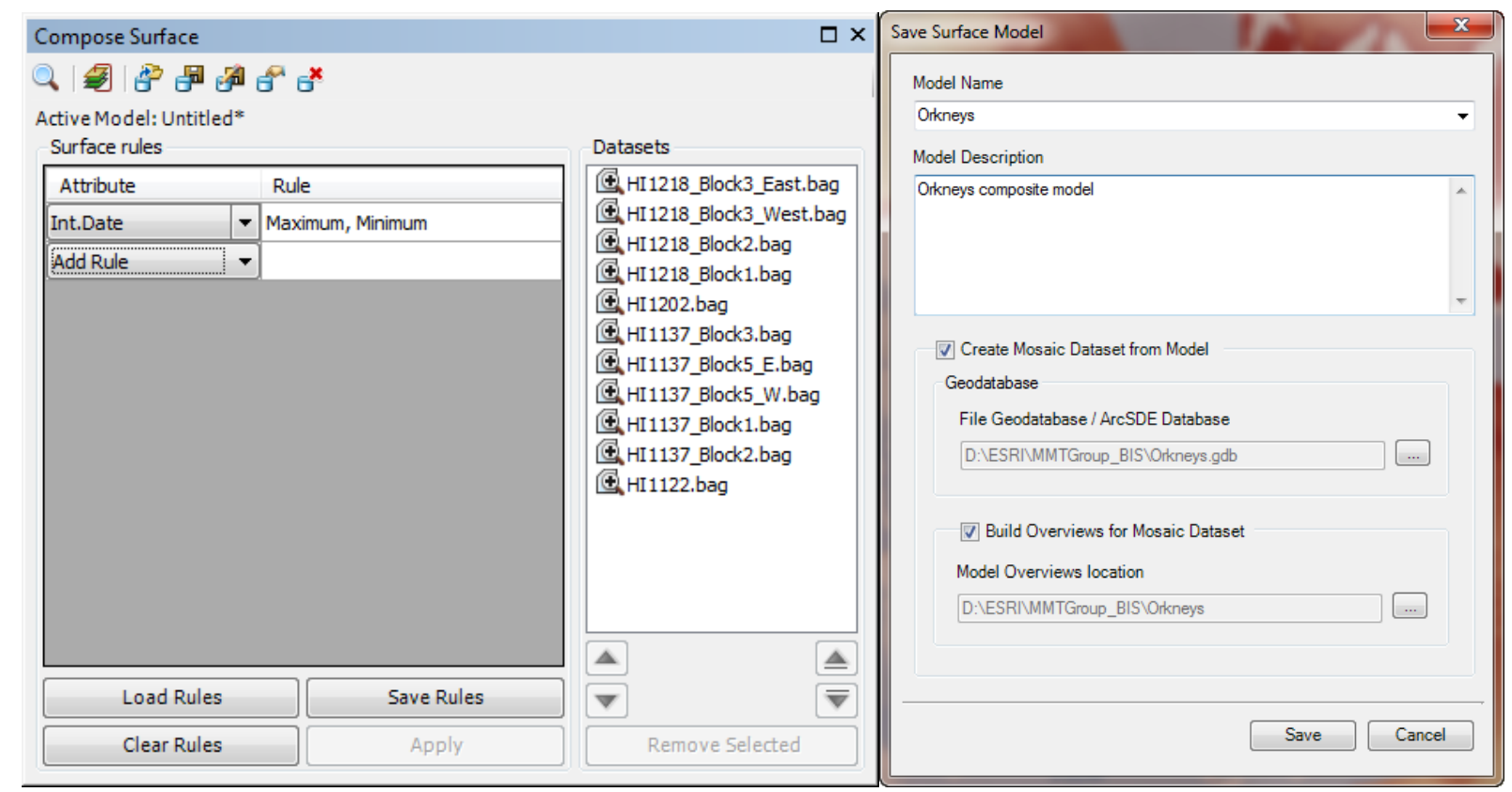

Figure 12: Creating a rule based on the most recent survey takes priority if they overlap and saving combined surface

Options are given to save the surface into the BIS geodatabase or create a separate geodatabase. The geodatabase just stores the image views seen in ArcMap and not the entire dataset.

Once the composite model has been formed the Arc Toolbox can be used on the model as if it were a normal ArcGIS raster product. In this case we are running the contour creation tool from the $3 \mathrm{D}$ Analyst toolbox over the entire dataset. To give you an indication of performance the Compose Surface took about 3 mins to run on 8.9 GBs of BAGs. The contour option then took about the same time to produce, in this case, contours at 1om intervals across the dataset. 


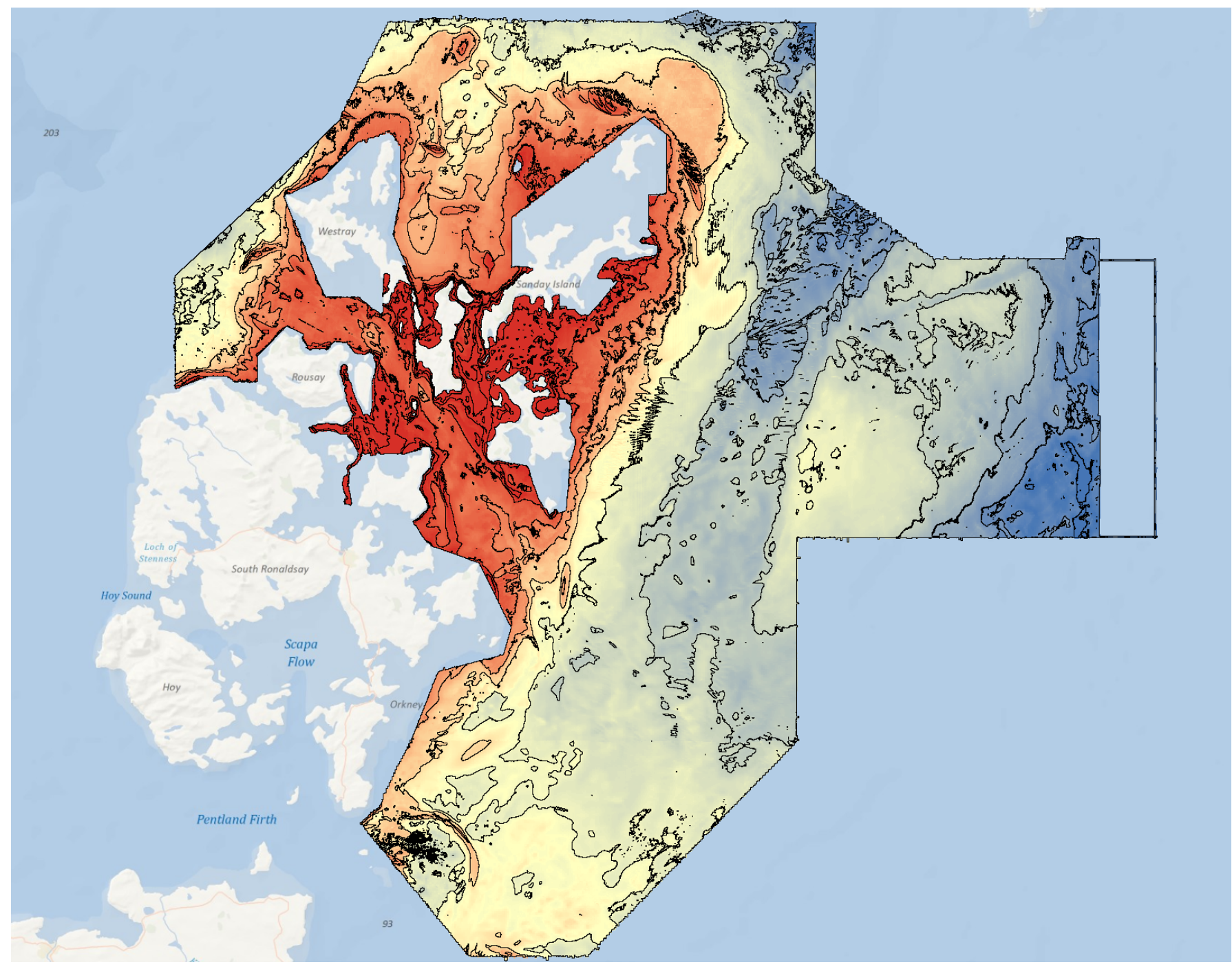

Figure 13: ArcMap showing the contours on top of the combined surface.

We find that increasingly our clients are requiring ArcGIS based products. One of the most recent of these has been the Seabed Survey Data Model that has been created by the OGP Geomatics Committee (what was the EPSG). This is a blank Esri geodatabase template with specific fields that can be populated by the results of a geophysical survey. This provides a controlled deliverable format that can also be used between Oil \& Gas clients if they need to share data. The template also provides a very convenient way for the Oil \& Gas companies to ingest the data into their Esri GIS systems. As we are already in ArcGIS with our bathymetric data and products it is trivial to populate the Seabed Survey Data Model.

\section{$\underline{\text { Future Uses }}$}

One of the main future uses we see is ArcGIS.com. This online web portal can be used both internally within the Group, to select clients and also some public access. The BIS includes a basic set of standards-based web services. These web services may be accessed directly or incorporated into composite services to deliver additional value to ourselves and our clients.

We can therefore host our clients data and provide them with an easy means of gaining access to their data and manipulating for their requirements. None of this requires the client to be GIS savvy and they can access the data from their desktops, tablet devices or smart phones.

We see another great benefit in the Esri Pipeline Data Model as a new method of delivering pipe and cable route and inspection surveys. By combining the pipeline survey surfaces into one high resolution combined surface, products can be created that transfer easily into the Esri Pipeline Data Model. 


\section{Conclusions}

The Esri ArcGIS for Maritime: Bathymetry solution is starting to revolutionise the way that MMT Group stores and accesses our bathymetric data. The quick, spatial access to our data coupled with the ability to combine survey datasets together is enabling us to speed up, not only our deliverable production but also our use of bathymetric data within the organisation, from initial tendering all the way through to final product.

With our use of ArcGIS.com it will enable our clients to use the full density products much more readily and without having to have a thorough GIS knowledge. This I think will change the way that clients perceive the data and will help in the shift from requiring deliverables based on older paper based products to new interactive digital products.

Most graduates from Geomatics based degrees come out with some ArcGIS knowledge. Very few come out with AutoCAD or other industry specific COTS products. By moving our deliverable production over to ArcGIS will reduce our training requirements and benefit new employees into understanding our work and the benefits to our clients.

Think spatial rather than flat! 